Pacific Journal of Mathematics

1-DIMENSIONAL PHENOMENA IN CELL-LIKE MAPPINGS ON 


\title{
1-DIMENSIONAL PHENOMENA IN CELL-LIKE MAPPINGS ON 3-MANIFOLDS
}

\author{
R. J. DA VERM AN
}

\begin{abstract}
Two 1-dimensional phenomena are studied. One resides, in the 3manifold domain of a cell-like map $/: M^{3} \longrightarrow Y$ and consists of an infinite 1-skeleton $X$ on which / is 1-1; if, in addition, the nondegen-

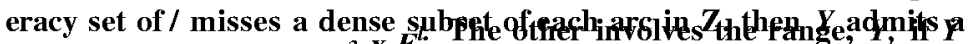
natural embedding in $\mathrm{A}^{3} \times \mathrm{E}^{\mathrm{h}}$

is a 3-manifold except possibly at points of a 1-complex F, topologically embedded in $Y$ as a closed subset, then / can be approximated by another cell-like map $p: M \longrightarrow Y$ whose nondegenecaqd sanhas embedding dimension $\leq 1$ and $/ \mathrm{x}$ Id: $M^{3} \times E^{r}$ approximated by homeomorphisms.
\end{abstract}

1. Introduction. Consider a proper cell-like surjective mapping /: $M$ - $Y$ denned on a 3-manifold $M$. This paper addresses the questions: Under what conditions can / be approximated by a cell-like mapping $F: M \longrightarrow Y$ for which each set $F^{\prime l(y)}$ is 1 -dimensional? Under what conditions can it be approximated by $F: M-Y$ such that the nondegeneracy set $N p$ of $F$ (deñned as

$$
\left.N_{F}=V\left\{F \sim^{l(y)} \backslash y e Y \text { and } F \sim^{l(y)} \text { is not a singleton }\right\}\right)
$$

has embedding dimension at most one (in the sense of Štan'ko [ $\left[\mathrm{St}_{\text {t }}\right.$ and Edwards [El])?

Several reasons can be adduced for interest in these matters. One simply is to improve known results about which spaces $Y$ are factors of some 4-manifold or, short of that, about which spaces $Y$ have a natural embedding in some 4-manifold (such as in $M \times E^{x}$ ). Another reason, part of a personal agenda not completely revealed here, is for use (to put it optimistically) in sought-for internal characterizations of those cell-like images $Y$ that are 3-manifolds, a problem in which map improvement techniques have been exploited with notable success by Edwards [E2].

Before stating the main results, we need certain fundamental definitions. A proper (surjective) map $p: M \longrightarrow Y$ defined on a manifold $M$ is said to have the Isotopy Disjoint Arcs Property (to be abbreviated as: Isotopy DAP) if for each pair of disjoint, locally flat arcs $a$ and 0 
in $M$ and for each open cover ${ }^{\wedge}$ of $Y$ there exists an isotopy ${ }^{*} F_{Z}$ of $M$ to itself such that

(i) $*$ Fo is the identity,

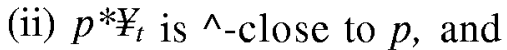

(iii) $/>¥,\left(<^{\star}\right) \mathrm{n} /^{\wedge}(0)=0$.

Similarly, $p: M-7$ is said to have the Homeomorphism Disjoint Arcs Property (abbreviation: Homeomorphism DAP) if, with the same data given above, there exists a homeomorphism $H: M \longrightarrow M$ such that $p H$ is ${ }^{\wedge}$-close to $p$ and $p H(a) \mathrm{n} />\#(/ ?)=0$.

For comparison, recall that a space $Y$ is said to have the Disjoint Arcs Property (DAP) if all pairs of maps $/, g$ of a 1 -cell $B^{l}$ into $Y$ can be approximated by maps $F, G$ having disjoint images. Generalized $n$-manifolds $(n \geq 3)$ invariably satisfy this DAP [D2], the weakest of the disjoint ares properties; in fact, should an infinite-dimensional cell-like image of an $n$-manifold exist, it would satisfy the DAP as well.

The focus throughout rests on the case $n=3$, the only dimension in which there is any doubt whether the stronger properties are satisfied, and the paper works around the following unresolved issue:

If $p: M-\quad Y$ is a cell-like map defined on a 3manifold $M$ such that each $p \sim^{l(y)}$ has a neighborhood that can be embedded in the 3 -sphere $\mathrm{S}^{3}$, does $p$ have the Homeomorphism (Isotopy) DAP?

The Homeomorphism DAP is useful, more so than the unadorned DAP, since maps $p: M \rightarrow Y$ satisfying the former can be approximated by comparable maps $/: M-Y$ for which each $f_{\sim} \sim^{l(y)}$ is 1-dimensional. This corresponds precisely to what can be done in higher dimensions, where $p$ can be approximated by a map / such that each $f^{l(y)}$ is $(n-2)$-dimensional, with $/$ attained to be $1-1$ on the union of the 1-skeleta of a preassigned sequence of triangulations of $M$ (see Proposition 2.4). Better yet, if $p: M \rightarrow Y$ is a cellular map with the Isotopy DAP, then $Y$ admits a natural embedding in the 4manifold $M x E^{x}$ (Corollary 3.5). Finally, if $p: M \rightarrow Y$ is a cell-like map such that each $p^{x(y)}$ has a neighborhood embeddable in $\mathrm{S}^{3}$ and if $Y$ has a closed subset $T$ homeomorphic to a 1-complex, where $Y-T$ is a 3-manifold, then $p$ has the Isotopy DAP and, furthermore, $p$. can be approximated by a cell-like map $F: M-Y$ such that (i) $\mathrm{i}^{7}$ is $1-1$ over $Y-T$ and (ii) $N p$ has embedding dimensiener ih thegorem whd de as a result, $Y x E^{l}$ is homeomorphic to $M \times E^{l}$, not all $p^{\prime l}(y)$ have neighborhoods embeddable in $S^{3}$ (Corollary 4.5). 
The results contained herein pertain to 3-manifolds $M$, whether compact or not. In the proofs, for simplicity, $M$ is usually presumed to be compact. Most arguments go through with little change beyond the epsilonic controls, which in general should be exercised by means of a positive-valued mapping rather than by constants. Significant exceptions occur when certain function spaces $M-\bullet[0,1]$ are considered; if $M$ is noncompact, one must use the limitation topology, described in [T], in order to be working with a Baire space, the essential item needed to validate the arguments presented.

The author wishes to express appreciation to J. J. Walsh for suggestions simplifying the proof of Theorem 3.1, to W. Jakobsche for several helpful conversations, and to the referee for catching a glaring oversight.

2. Disjoint ares properties. The chief concern in this paper will be with cell-like maps defined on 3-manifolds $M$, for the ones defined on higher dimensional manifolds are known to satisfy the strongest possible disjoint ares property. The argument is little more than reapplication to a far simpler situation of an idea used repeatedly by Edwards [E2].

PROPOSITION 2.1. Each proper cell-like map p: $M \rightarrow Y$ defined on an $n$-manifold $M(n>4)$ has the Isotopy DAP.

Proof. Given $a$ and $f t$ in $M$, there is a controlled homotopy between $p \backslash a \mathrm{U} f t$ and some new map /: a U j $8-\gg \mathrm{F}$ where /(a) and /(/?) are disjoint [D2]. The homotopy lifts (approximately) to a homotopy in $M$ between the inclusion and some locally flat embedding $F: a j$ $f t-* M$, which in turn can be covered (approximately) by a controlled isotopy of $M[\mathbf{B K}]$.

Let $G$ be a cell-like use decomposition of a 3-manifold $M$ with decomposition map $i t: M \longrightarrow M / G$. In the space of all maps $M \longrightarrow M / G$ we identity

$$
\begin{aligned}
\% f & =C \backslash n h \mid h: M \longrightarrow M \text { is a homeomorphism }\} \text { and } \\
J^{\prime \prime} & =C l\left\{n d \mid 8: M-M \text { is isotopic to } l d_{M}\right\}-
\end{aligned}
$$

An infinite l-skeleton in a 3-manifold $M$ is the union of the 1skeletons from a sequence $T j$ of triangulations of $M$, where $\operatorname{mesh}(7\}) \rightarrow$ 0 as $/ \rightarrow$ oo. 
PROPOSITION 2.2. If.G is a cell-like decomposition of a 3-manifold. $M$ and $\mathrm{S}^{\wedge} 1^{\prime}$ is an infinite lskeleton in $M$ such that $n: M \stackrel{3-M}{M} G$ is 1-1, then $n$ has the Isotopy DAP.

Proof. Consider disjoint ares $a$ and /? locally flatly embedded in $M$. Measure the distance $S$ between $a$ and $/$ ?. Fix e $>0$ and restrict $S$ so the image under $n$ of any $S$-subset of $M$ has diameter less than $s$. Determine a triangulation $T$ of $M$ for which $T^{\wedge} \mathrm{c} \mathrm{X}^{(1)}$ and mesh $T<S$. Construct a pseudo-isotopy $y / s: M \longrightarrow M$ (a homotopy starting at Idjv/ with each level $i / / s, s<1$, being a homeomorphism) moving

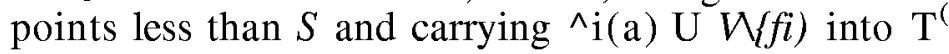
$\wedge \mathrm{i}(/ ?)=0$, so $7 \mathrm{i}^{\wedge} \mathrm{i}(\mathrm{a}) \mathrm{n} n y / \mathrm{i}(P)=0$. Choose a value $\mathrm{w}$ of 5 close to 1 for which $n y / u(a) \mathrm{n} n y / u(P)=0$ - The isotopy $\wedge$, restricted to run between 0 and $u$, shows $n$ has the Isotopy DAP.

D

LEMMA 2.3. If it: $M-* M / G$ is a cell-like decomposition map defined on a 3-manifold $M$ and $p €<\% *$, then

$$
\% f=c \backslash p h \vee h: M \longrightarrow M \text { a homeomorphism\}. }
$$

The argument rests on a simple observation: $p(n h, p)<e$ if and only if $p\left(n, p h^{l)}<e\right.$.

PROPOSITION 2.4. Suppose $G$ is a cell-like use decomposition of a 3manifold $M$ and suppose $\mathrm{Z}^{\wedge}$ is an infinite l-skeleton in $M$. Then the following statements are equivalent:

(A) $n: M \rightarrow M / G$ has the Homeomorphism DAP.

(B) $n$ can be approximated, arbitrarily closely, by maps $p$ e 3? such that $p \mathrm{~V}, W$ is $1-1$.

(C) There exists peJT such that pll('> M 1-1.

Proof. The implication (A) $=>$ (B) involves an application of the Baire Category Theorem. Enumerate all pairs $A$; of disjoint 1-simplexes $(a, T)$ in $T L^{\wedge}$ or any of the successive barycentric subdivisions of its simplexes. Let J\#J denote the subset of \%? deñned as:

$$
j^{*}{ }_{i}=\{\text { pe2'lp(a)n } P\{T)=O\},
$$

where $A t=(a, \mathrm{~T})$. By hypothesis, sfi is dense in $\% *$, and by a standard argument, as in [HW], it is open there. The Baire Category Theorem ensures the existence of a map $p: M, \rightarrow M / G$ in $f s / j$ close to $n$. Finally, to any distinct points $x, y \mathrm{eX}^{\prime 1}$ there corresponds an index $i$ such that $x e a$ and yet, where $A_{t}=(a, \mathrm{~T})$; hence, $p \backslash 2^{(1)}$ is $1-1$. 
That $(\mathrm{B})=>(\mathrm{C})$ is obvious.

For the remaining implication, $(\mathrm{C}) \Rightarrow(\mathrm{A})$, the hypothesis and the comment about the proof of Lemma 2.3 establish the existence of a homeomorphism $h: M \rightarrow M$ with $p^{\prime}=p h^{x}(£ / 3)$-close to $n$. Then $p^{\prime}$ is 1-1 on the infinite 1-skeleton $h\left(L^{\wedge}\right)$. Given disjoint arcs a and $P$ locally flatly embedded in $M$, apply Proposition 2.2 to obtain a homeomorphism $h \backslash M \longrightarrow M$ such that $p^{\prime} h^{\prime}$ is within e/3 of $p^{\prime}$ and $\left.p^{\prime} h^{\prime}(a) \mathrm{n} p^{\prime} h^{\prime} i f i\right)=0$. Finish this off by putting Lemma 2.3 into operation to determine another homeomorphism $H: M \rightarrow M$ such that $n H$ is at least (e/3)-close to $p^{\prime} h^{\prime}$ and, moreover, $n H(a) C n H(j i)=$ 0 . Then $H$ has the desired effect on $M$ and $n H$ is $£$-close to $n$.

A characterization of the Isotopy DAP can be derived with a similar argument.

PROPOSITION 2.4'. For $M, G$ and $I t^{1)}$ as in Proposition 2.4, the following statements are equivalent:

(A) $n: M-M / G$ has the Isotopy DAP.

(B) For each $e>0$ there exists an isotopy $* \mathrm{~F}_{\mathrm{f}}: M \rightarrow M$ definedfor $t$ e $[0,1)$ such that $p(n, i W t)<\mathrm{e} / \mathrm{w} \ll / / 1 €[0,1)$, f/ze limit $p$ ofiC $\#_{t}$ as $t \rightarrow 1^{*} \mathrm{w}$ continuous, and $p \backslash \mathrm{Z}^{\wedge} \dot{\mathrm{w}} 1-1$.

(C) 77zere exw? $\mathrm{p} € S$ and an isotopy $* \mathrm{~F} ;: M \longrightarrow \mathrm{M}$ defined for $t \mathrm{G}[0,1) \operatorname{swc} / \mathrm{z}$ f/za? $\mathrm{p}=\operatorname{Iim} 7 \mathrm{r}^{\wedge}\left(\mathrm{a}^{*} t-\gg 1\right)$ anrf $\mathrm{p} \mid \mathrm{IO} \dot{\mathrm{w}} 1-1$.

The preceding expose invariance properties.

COROLLARY 2.5. Let $G$ be a cell-like use decomposition of a 3-manifold $M$. Then $n: M-* M / G$ has the Homeomorphism DAP (Isotopy $D A P)$ if and only ifeach $p € \% *$ (each $p$ e S) does.

Proof. When $n$ has the Homeomorphism DAP, then certainly so does each member of $\{n h \backslash h: M \rightarrow M$ a homeomorphism $\}$ which is dense in $\mathrm{J}^{\prime \prime}$. On any given infinite 1 -skeleton $\mathrm{I}^{(1)},\{q € \% \mathrm{q} q$ is $1-1$

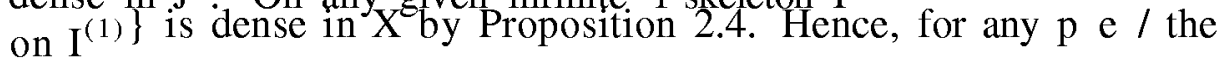
combination of Lemma 2.3 and a second application of Proposition 2.4 implies $p$ has the Homeomorphism DAP.

The next result can be proved using the Baire Category Theorem and an adaptation of the proof of Proposition $2.4\left(2.4^{\prime}\right)$. It reveals that the restriction in the definitions of Homeomorphism DAP and Isotopy DAP to locally flat arcs $a$ and $/ ?$ is dispensible. Details are left to the interested reader. 
PROPOSITION 2.6. Suppose $G$ is a cell-like use decomposition of a 3-manifold $M$ such that $n: M \rightarrow M / G$ has the Homeomorphism DAP (Isotopy DAP), and suppose $Y$ is a a-compact set in $M$ having embedding dimension $<1$ Then $n$ can be approximated by a map $p € \%$ ? $\left\{p \mathrm{e} \mathrm{J}^{2}\right)$ such thât $p \backslash Y$ is 1 -1.

PROPOSITION 2.7. Suppose $M$ is a 3-manifold, $\mathrm{I}^{(1)}$ is an infinite 1-

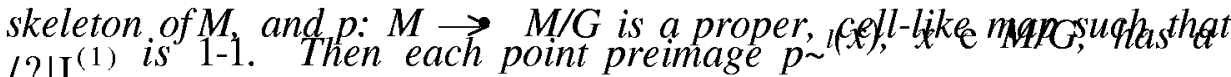
neighborhood in $M$ that embeds in $S^{3}$.

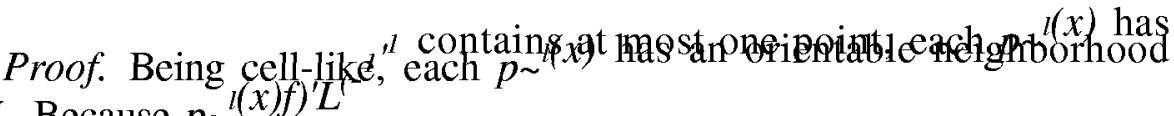
W. Because $p \sim^{l(x) f)^{\prime} L}$ embedding dimension $\leq 1[\mathrm{El}]$. Therefore, there exists a cube-with-
handles $H$ in $W$ containing $p^{x}(x)$ in its interior [M2].

3. Trivially extended decompositions. Given a cell-like use decomposition $G$ of a 3-manifold $M$, where $M$ is $G^{\prime}$ denithed depammosition in $M \times E^{l}$, its trivial extension $G$ into elements of $G$ and the sip $t e t h G_{\text {rom }}^{T}$ to denote the decomposition we will use $n^{T:}$

map associated with this trivial extension.

THEOREM 3.1. Suppose $M$ is a 3-manifold, $\mathrm{E}^{\wedge}$ is an infinite 1skeleton in $M$, and $G$ is cell-like use decomposition of $M$ such that $n \mid Z O$
$G$

Proof. Express $\mathrm{E}^{\wedge}$ as the union of 1-complexes $T J{ }^{1}{ }^{1)}$, where $T j$ denotes some triangulation of $M$ having mesh less than $l / i$. Thenestrateser

is to reorganize $G^{T,}$ via a homeomorphism $\mathrm{O}$ of $M x$
commuting with the projection $M x E^{x}-M$, so that

(1) each nondegenerate element $<b\left(g^{*}\right), g^{*} \in G^{T}$, lies in a level $M x\{t\}$ of $M x E^{l,}$

(2) the decomposition $\&^{1}$ of $M$ induced by $\mathrm{O}\left(\mathrm{G}^{\mathrm{r}}\right)$ on any slice $M \times\{t\}$ is 1-dimensional, where the nondegenerate elements of $S ?^{1}$ are $\left\{g e H_{G}\langle t>(g \times\{0\}) \subset \mathrm{M} \mathrm{x}\{;\}\}\right.$, and

(3) for a dense set $3 i$ of values $d €(0,1)$, the decomposition " $3^{d}$ is of (source) embedding dimension $\leq 1$. According to [DP2], the decompositions " $\S^{d} x E^{l}$ will be shrinkable decompositions of $M x E^{l}$ for all $d € 2$, and by [DPI] the trivial 
extensions of each ${ }^{\wedge^{\prime}}$ over $M x E^{l}$ will be shrinkable. With that data, the Theorem of [DPI] will attest $\mathrm{O}\left(\mathrm{G}_{\mathrm{r}}^{\mathrm{r}}\right)$ is shrinkable and, hence, the same is true of $G^{T}$ itself.

The homeomorphism $\mathrm{O}$ will be denned by means of a map $p: M / G$ - $[0,1]$ and will be specified as

$$
\%((x, s))=\{x, s+/ u c(x)) .
$$

It is an exercise that such a map $\mathrm{O}^{\wedge}$ is a homeomorphism of $M x E^{l}$ onto itself, and it should be clear why $\mathrm{O}^{\wedge}$ then must satisfy (1) above.

It is useful to note $M / G$ is 3-dimensional, by the result of Kozlowski and Walsh [KW] (see also [W]). Since $S^{(1)}-N_{G}$ is dense in $M, \operatorname{dim} N o \leq 2$ and, as a consequence, $\operatorname{dim} 7 \mathrm{r}\left(\mathrm{iV}_{\mathrm{G}}\right) \leq 2$, because $n$ cannot raise dimension.

We pause to establish two facts of a dimension-theoretic nature.

LEMMA 3.2. Let $W$ denote the space of maps $X \rightarrow I=[0,1]$, endowed with the sup-norm metric, defined on a separable metric space $X$, and let $Z$ denote $a^{\wedge}$-dimensional $F_{a-s e t}$ in $X$. Then

$$
j^{*}=\{X e W X \text { is } 1-1 \text { onZ }\}
$$

is a dense Gg-subset of W.

Proof. Write $Z$ as the countable union of sets $Z$, $(/=1,2, \ldots)$ closed in $X$, and set

$$
\&(i . j)=\{X<E W X Z I \text { is a }(1 / 7>\operatorname{map}\} .
$$

A combination of techniques from [HW] and a controlled version of the Borsuk Homotopy Extension Theorem yields that $\wedge(i, j)$ is dense in $W$. Clearly $s f=f l W(i, j)$. Application of the Baire Category Theorem completes the proof.

LEMMA 3.3. If $N$ is a $k$-dimensional $F_{a-s e t}$ in the space $X$ of Lemma 3.2 , where $0<k<00$, then $X$ contains a dense $G \$$-set $s /$ such that $\operatorname{6im}\left\{N r\left(X_{\sim} x(t)\right)<k\right.$ for each tel and $\lambda \in \mathscr{N}^{\prime}$.

Proof. By [HW, pp. 30-32], iV can be expressed as $P$ U Z, where

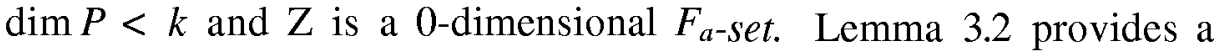
dense Q-set $\mathrm{J} /$ ' in $W$. where each $X £ s f^{\prime}$ is $1-1$ on $Z_{\text {. Then for }}$ all $t € l, N \cap X \sim^{l}(t)$ lies in $P$ plus at most 1 point of $Z$, yielding $\operatorname{dim}\left(\mathrm{iVnA}{ }^{\prime 1}(0)<^{\wedge}[\mathrm{HW}\right.$, p. 32]. 
Completion of Proof of Theorem. 3.1 Now let $W$ denote the space of all maps $f x: M / G \longrightarrow[0,1]$, with the sup-norm metric. Express $n(£(') \mathrm{n} N Q)$ as the union of compact 0 -dimensional sets $\mathrm{Q}$. $(k=$ $1,2, \ldots)$. By Lemma 3.2, $W$ contains a dense $\mathrm{Gj}$-set

$$
\mathscr{A}=\left\{\lambda \in \mathscr{E} \mid \lambda \text { is } 1-1 \text { on } \bigcup C_{k}\right\} .
$$

and by Lemma 3.3, $W$ contains another dense $\mathrm{G}^{\wedge}$-set $\$ f^{\prime \prime}$ such that $N_{G n k^{l}}{ }^{l}(t)$ is 1-dimensional, for all $k e s Z^{\prime}$ and $t e[0,1]$. The Baire Category Theorem provides $/ u$ e $\left\{\$ ? C\left(s /{ }^{\prime}\right)\right.$, which gives rise to the desired homeomorphism $\mathrm{O}^{\wedge}$ satisfying Conditions (1), (2) and (3) above.

THEOREM 3.4. Let $G$ be a cell-like decomposition of a 3-manifold $M$ such that $n$ has the Isotopy DAP and

$$
\left\{x € M / G \mid n^{\alpha(x)} \text { is cellular in } M\right\}
$$

is dense in $M / G$. Then the trivial extension $G^{T}$ of $G$ to $M \times E^{l}$ is shrinkable.

Proof. The idea is to produce another cell-like map $p: M-* M / G$ from the limit as $t \rightarrow 1$ of $n^{\wedge} t$, where $4 \mathrm{~V} M \rightarrow M$ is an isotopy beginning at $\mathrm{Id}^{\wedge}$ and defined for $t$ e $[0,1)$, with $p$ 1-1 on some infinite 1-skeleton $Z^{(1)}$ such that

$$
\operatorname{dim}\left(\Sigma^{(1)} \cap N_{p}\right) \leq 0
$$

It is convenient to regard $* \mathrm{Fi}: M \longrightarrow M$ as a set-valued function (or, relation) determined by the limit of $\wedge^{\wedge}$ as $t \rightarrow \vec{C}^{T}$ as 1 . To each $x G M$ there will correspond $g_{x} G G$ such thar(NFix $E^{l} E^{l} / G_{x}^{T}$. We will prescribe a new cell-like map $p^{T:^{x}} M x$

$$
p^{T}(\langle x, s\rangle)= \begin{cases}\pi^{T}(\langle x, s\rangle) & \text { if }|s| \geq 1 \\ \pi^{T}\left(\left\langle\Psi_{1-|s|}(x), s\right\rangle\right) & \text { if }|s| \leq 1\end{cases}
$$

Then $p^{T}$ will behave like $/ ?$ on $M$ x $\{0\}$, and Theorem 3.1 will imply the shrinkability of the decomposition induced by $\beta^{T}$ show The shrinkability of $G^{T}$ will follow, either by redesigning $p$

made arbitrarily close $_{\mathrm{a}} \mathrm{or}^{T}$ or by noting from the foregoing that $\left(M \times E^{X}\right) / G^{T}$

imation Theorem [Q, Corollary 2.6.2] (a nice exposition of Quinn's result can be found in $[\mathrm{A}]$ ). 
In order to obtain $p=1 \mathrm{im} \mathrm{m}^{\wedge} \wedge$, name an infinite 1 -skeleton $\mathrm{Z}^{\mathrm{1}^{1}}$ in $M$ and a countable set $\mathrm{Z}=\{z, \backslash i=1,2, \ldots\}$ c $\mathrm{Z}^{\wedge 1}$ ) such that $\mathrm{Z} \mathrm{n} a$ is dense in each arc $a c Z^{(1)}$. Define

$$
\wedge=\left\{\text { pe } J^{\prime} \backslash \text { diamp- }^{l}(Z J)<l / i\right\} .
$$

Not only is $J ? i$ open and dense in $J^{\prime}$, each $n h € J^{\wedge}\left(h: M-^{\wedge} M\right.$ a homeomorphism) is close to some $p \mathbf{e}^{\wedge}$ under a short isotopy, obtained by first pushing $\mathrm{z},-$ to a point in some cellular preimage and then isotopically shrinking that preimage to small size. Consequently, we can build an isotopy $¥,: M \rightarrow M$, defined for $t €[0,1)$, such

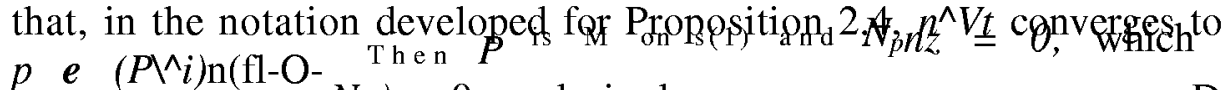
reveals $\operatorname{dim}\left(\mathrm{E}^{(1)} \mathrm{n} N p\right)<0$, as desired.

COROLLARY 3.5. IfG is a cellular use decomposition of a 3-manifold

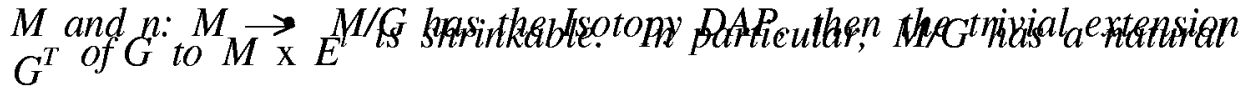

embedding in $M \times E^{\text {. }}$

Heretofore the strongest result comparable to Corollary 3.5 reached the same conclusion for cell-like decompositions $G$ of $M$ such that $\operatorname{dim}^{\wedge}(G) \leq 1$ [DPI, Corollary 8].

4. Decompositions of embedding dimension $\leq 1$. Several years ago the author asserted [D1, p. 135], without proof, that the following was true. This section sets forth details.

THEOREM 4.1. Suppose $G$ is a cell-like use decomposition of a 3manifold $M$ for which the singular set, $S(M / G)$ ), lies in a (-complex $\mathrm{F}$ embedded in $M / G$ as a closed subset and suppose each $g$ e $G$ has a neighborhood $U_{g}$ embeddable in $S^{3}$. Then $n: M \longrightarrow M / G$ has the Isotopy DAP; furthermore, arbitrarily close to $n$ is a map / ) G / such that $N_{p}$ has embedding dimension $\leq 1$.

What is needed to establish Theorem 4.1 is a controlled arc-pushing property, describing how to divert a given arc in $M$ away from $n \sim^{l(1)}$ by means of a motion whose image under $n$ is small. McMillan (cf. [M1] [M2] [M3]) and also Row [R] have studied less strictly controlled arc-pushing properties extensively and have demonstrated the close connection to cubes-with-handles properties. Stated next is a controlled cubes-with-handles result that leads to a useful arc-pushing property, presented in Proposition 4.4. 
LEMMA 4.2. Suppose $U$ is an open subset of $E^{3}$ with connectedfrontier, $p: X-* E^{x}$ is a proper cell-like map defined on a closed subset

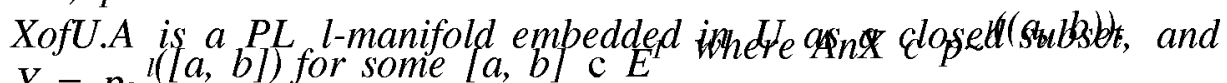
$Y=p \sim$

Then there exist a cube-with-handles $H$ and a compact 2-manifold $F$ in $d H$ satisfying:
(1) YclntHcHcU,
(2) each component of $F$ is a 2-cell,
(3)IntFDXndH, and
(4) $F D A=O$.

Proof. Being contractible in $U, X$ has a neighborhood $U^{*}$ contractible in $U$. The cell-like set $Y \subset X$ lies interior to some cubewith-handles $H^{*}$ in $U^{*}$ [Ml, Theorem 2'].

The argument involves modification of $H^{*}$ by simple moves, in the sense of McMillan [M3]. The first step causes the boundary of the resulting manifold $K$ to meet $X$ in a finite union $F^{*}$ of pairwise disjoint 2-cells in $d K-A$. The second, entailing further alteration to $K$, resurrects a cube-with-handles.

To get started, determine a PL manifold neighborhood $N$ of $X \mathrm{n}$ $d H^{*}$ in $d H^{*}-A$ such that each loop in $\operatorname{Int} N$ is null homotopic in $\left(U^{*}-\left(Y l i A U d H^{*}\right)\right) \mathrm{U}$ IntiV (this can be arranged by locating TV so close to $X$ that loops in Int iV are homotopic there to loops very near $X$, which then can be contracted missing $Y U A$ and striking $d H^{*}$ only inside $N$ ).

If every component of $N$ should happen to be included in some disk in $d H^{*}-A$, these disks easily could be cut apart to form the required $F^{*}$. Otherwise, one prepares to make a simple move by identifying a simple closed curve / in Int $N$ not bounding a disk in $N$ (equivalently, / not contractible in $N$ ). There exists a map

$$
f i: B^{2^{\wedge}\left(U^{*}-\left(Y u A U d H^{*}\right)\right)} \mathrm{U} \text { IntiV }
$$

tracing out / homeomorphically along the boundary. Assuming $\backslash i$ to be PL and in general position with respect to $d H^{*}$ one can find a disk with holes $D$ in $B^{2}$ such that $n(d D) \mathrm{c} N$, fi(Int $\left.D\right) \mathrm{n} N=0$, and on precisely one component $C$ of $d D j n \backslash C: C \rightarrow \operatorname{Int} N$ is homotopically nontrivial. As a result, one can regard /i as defined on a disk $D$ without holes, where $C=d D$. Invoke the Loop Theorem to obtain a PL embedding ; $\mathrm{U}^{*}$ of $D$, either into Int $N u\left(\operatorname{Int} H^{*}-(Y u A)\right)$ orintoIntiVU $\left(U^{*}-\left(A \mathrm{U} H^{*}\right)\right)$, with $n^{*}(d D) \mathrm{C}$ Int $N$ in homotopically nontrivial fashion. Write $n^{*}(D)$ as $B$ and thicken it to a 3-cell $B \times /$ in either 
$\left.H^{*}-(Y l) A\right)$ or in $U^{*}-\left(A u \operatorname{lnt} H^{*}\right)$ with $(B \times I) n d H^{*}=(d B) \times / \mathrm{c}$

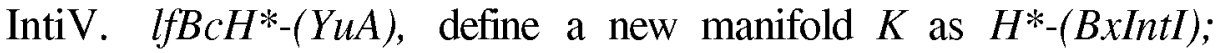
otherwise, define $K$ as $\left.H^{*} l\right)(B \times I)$.

No matter which side of $d H^{*}$ includes $B$, let $N_{K}$ denote (iV n $d K$ ) U $(5 \mathrm{x} d l)$. Then $\mathrm{X} \mathrm{n} d K \mathrm{c} \mathrm{iV}>$, a 2-manifold in $d K-A$. Observe how every loop in $K$ contracts in $U$, for when $K$ is larger than $H^{*}$ each loop there is homotopic through $K$ to one in $H^{*}$. Also observe that loops in $N K$ contract in $\left[U^{*}-(Y \mathrm{U} A \mathrm{U} d K)\right] \mathrm{U}$ Int $N K$, by performing an initial homotopy through $N K$ into $N$.

Consequently, upon verification of the claim below, Lemma 4.2 will follow from the forthcoming Lemma 4.3.

Claim by a finite number of simple moves $H^{*}$ can be transformed to a PL 3-manifold $K$ with $Y$ c Int $K$, all loops in $K$ null homotopic in $U$, and $X n d K$ contained in a finite union $F^{*}$ of pairwise disjoint 2-cells in $d K-A$.

Proof of the Claim. Define the complexity $q(P)$ of a compact 2manifold with boundary $P$ as

$$
q(P)=(\text { number of components of } d P)-x(P)
$$

( $X=$ Euler characteristic). Provided no component of $P$ is a 2 -sphere or a projective plane, (i) $q(P) \geq 0$ and (ii) the components of $q(P)$ are all 2-cells if and only if $q(P)=0$. In the situation at hand, ng component of iV or $N K$ can be a projective plane, because $U \mathrm{c} E^{2}$, and by construction no component of $N$ is a 2-sphere so the same holds for $N K$. Hence, the claim follows from the straightforward verification (in the terminology used for the elementary modification above) that $q\left(N_{K}\right)<q(N)$.

LEMMA 4.3. Under the hypotheses of Lemma 4.2, let Kbea compact $P L$ 3-manifold such that $Y \mathrm{c}$ Int $K \mathrm{c} K \mathrm{c} U$ and each loop in $K$ is contractible in $U$, andlet $F^{*}$ be afinite union of pairwise disjoint 2 -cells in $d K-A$ with $\ln \backslash F^{*}$ DXndK.

Then there exist a cube-with-handles $H$ and a compact 2-manifold $F$ in $(d H)-A$ satisfying:
(I) $\mathrm{KCHCU}$,
(2) each component of F is a 2-cell, and
(3)IntFDXndH.

Proof. Except for the part about the 2-cells $F^{*}$ and $F$, the argument is given in [Ml, Lemma 1]. Following McMillan's procedure, every 
time we add a thickened disk to $K$ or delete one from $K$, we operate in the complement of $F^{*}$. This procedure involves compressing $d K$ with a succession of compressing disks, some of which compress (initially) to the outside of $K$ and some to the inside.

It is easy to adjust so those compressing to the outside pass nicely through thickenings of earlier compressing disks. The boundary of the resulting cube with handles $H$ is a subset of $d(K \backslash J(V E j \mathrm{x} /))$, where $E_{t}$ is a disk with holes obtained from the rth outside compressing disk by deleting its intersection with the thickenings of the earlier compressing disks, the $E j \times$ / are pairwise disjoint, their interiors miss $K$, and $E i$ x / meets $d K$ along $d E i$ x / c $d K$. [Remark: if no boundary of an outside compressing disk ever separates $d K$, then $H$ actually equals $K u d J E j X l)$; generally $H$ is determined by an outermost component of the boundary just named.] We conclude by showing how to adjust the relevant cores $V E_{t} \times\{0\}$ so they lie in $U-(A \mathrm{U} X \mathrm{U} \operatorname{Int} K)$.

To simplify notation identify $£$, with $E i \times\{0\}$ and write $\mathrm{Q}=\bigvee E j$. Thus, $Q$, is a compact (disconnected) planar surface in $U$ for which $Q n K=d Q c d K-\left(A \backslash F^{*}\right)$. Each $d E i$ has a distinguished component $\mathrm{Li}$-namely, the boundary of the larger compressing disk. The adjustment amounts to building (1) another compact planar surface $Q !=\mathrm{U} E^{\prime} j$, where Q' has the same number of components (the sets $E$ ) as $\mathrm{Q}, L_{t} \mathrm{c} E$, and $Q^{\prime} \mathrm{n} K=\mathrm{Q}^{\prime} \mathrm{n} d K=d Q^{\prime}$, and (2) a corresponding finite union $F^{\prime}$ of pairwise disjoint disks with

\section{I n i i c Int F' $\mathrm{c} \mathrm{f}^{\prime} \mathrm{c}($ IntQ') - $A$.}

Here $Q, \mathrm{n} Y=0$. Choose a neighborhood $W$ of $X-Y$ in $U-[A \mathrm{U}$ $\left.\left(d K-\operatorname{Int} F^{*}\right)\right]$, and find a smaller neighborhood $W$ such that loops in $W$ are contractible in $W$. In case $Q, \mathrm{n} X$ is not contained in a finite union of disks in Q, some simple closed curve / in, say, $£$; $\mathbf{n} W$ must separate two components of $d E i$ in $\mathrm{Q}$. Let $\mathrm{i}$ ? denote the component of $E j-J$ containing L,: One can use $i t$-properties of the inclusion $W \rightarrow W$ to define a natural map of $R \mathrm{U}$ disk into $\mathrm{i}^{\wedge} \mathrm{UfF}$ and then can apply the proof of a generalized Dehn's lemma due to Shapiro and Whitehead [SW] (or the controlled version of Dehn's lemma by Henderson $[\mathrm{H}]$ ) to find a disk with holes $Z$, having fewer holes than $E i$ such that $L i \mathrm{c} d D j \mathrm{c} d E$, and A c $E_{i} \bullet \mathrm{u} \mathrm{W}$. Because the last condition forces $£>\mathrm{n} d K \mathrm{c}$ IntF*, one can improve $D$; by trading disks between $D_{t}$ and $\mathrm{F}^{*}$ to make $D_{t} n K=D_{t} n d K=d D_{t}$. Repeating as often as necessary, one eventually will produce such à compact planar surface $£ 1 *$ for which $\mathrm{Q} * \mathrm{nX}$ is contained in a finite union $\mathrm{A}$ of pairwise disjoint 
disks in IntQ*. Should some component $B$ of $\mathrm{A}$ then meet $A$, one can decrease the size of $B$ to avoid $A$ unless $B C X$ (note: $B n X c X-Y$ ) separates $d B$ from a point of $B \mathrm{n} A$, but then more or less as before Dehn's Lemma (usual form) yields a map

$$
y /: A^{\wedge} \quad U \quad-\left[A u\left(d K-\ln t F^{*}\right) \Lambda\left(Q^{*} \quad-i n t A\right)\right]
$$

which reduces to the identity on $d A$ and is 1-1 on each component of A. Again, disk trading gives an embedding

$$
¥: \mathrm{A}->U-\left[A \mathrm{U} K \mathrm{U}\left(\mathrm{Q}^{*}-\operatorname{Int} \mathrm{A}\right)\right] \text {. }
$$

Set $Q !^{\prime}=\left(\mathrm{Q}^{*}-\mathrm{A}\right) \mathrm{u} ¥(\mathrm{~A})$ and $\mathrm{i} ?^{\prime}={ }^{*} \mathrm{~F}(\mathrm{~A})$, to complete the description of the modifications to $\mathrm{Q}$ and construction of disks $F^{\prime}$.

Finally, the compact 2-manifold $F$ called for in Lemma 4.3 is $F^{\prime} \mathrm{U}$ $\left(F^{*} \mathrm{n} d H\right)$.

PROPOSITION 4.4.Let Gbe a cell-like decomposition of a 3-manifold $M$ satisfying the hypotheses of Theorem 4.1, A a PL arc in $M$, and $V$ a neighborhood of TI $X T 1(A)$. Then for each $\mathrm{e}>0$ there exists a homeomorphism $h$ of $M$ onto itselfsuch that:

(1) $p(n(x), n h(x))<$ efor each $x$ e $M$,

(2) $h$ moves no point of $M-V$, and

(3) $n h(A) \mathrm{nT}=0$.

Proof. First modify $A$ slightly to ensure $X \mathrm{n} d A=0$. Afterwards choose a finite collection of points $Q \backslash, \ldots, q^{\wedge}$ separating $T$ so that, for any component $C$ of $T-\mathrm{U}\{<7 /\}$ whose closure meets $n(A)$,

$C$ is homeomorphic to $E^{\prime \prime}$

$\operatorname{diamC}<\mathrm{e} / 2$,

$\mathrm{r}^{\prime}(\mathrm{ClC}) \mathrm{cK}$ and

$7 \mathrm{r} \sim(\mathrm{ClC})$ has a neighborhood embeddable in $E^{3}$.

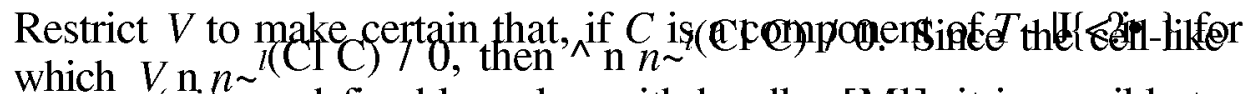
sets $n \sim x(q i)$ are defined by cubes-with-handles [Ml], it is possible to find a PL homeomorphism $h$ of Af to itself such that

$$
\begin{aligned}
& p(n(x), n h \backslash(x))<e / 2, \\
& h i \text { is fixed outside of } V, \text { and } \\
& q_{i} \notin \pi h_{1}(A) .
\end{aligned}
$$

Consider those components $C$ of $T-\mathrm{U}\{\# /\}$ intersecting $n / \mathcal{A} A)$. The same process will take place near each, so for simplicity assume 
there to be only one such component $C$. Construct an open set $U$ in $V$ containing $n \sim^{l(C)}$ and having connected frontier, with $U$ embeddable in $i^{3}$ and with $\operatorname{diam} n(U)<e / 2$. Apply Lemma 4.2 to find a cubewith-handles $H$ with $U D H \mathrm{D}$ Int $H D h \backslash(A) \mathrm{n} n_{\sim^{x}}(C)$ and also a finite union $F$ of pairwise disjoint disks in $d H-h \backslash(A)$. whose interiors cover $d H \mathrm{n} n \sim^{i}(\mathrm{C})$. Specify a PL spine $L$ of $H$ to which $H$ collapses, with $L$ consisting of a bouquet of circles plus some arcs, a distinct arc from the bouquet to each of the components of $F$. Finally, determine

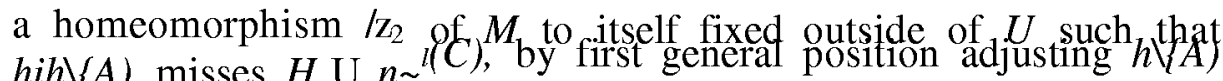
to avoid $L \mathrm{U} F$ and then exploiting the regular neighborhood structure of $H$ relative to $L$ to do the rest. The composition $h=\square 221$ has the desired effect.

Proof of Theorem. 4.1 Based upon Proposition 4.4 and the nowtypical approximation methodology, one can produce a new cell-like map $p: M \rightarrow M / G$ near $n$ in $<y$ (in fact, with $p=\operatorname{limn}\left\langle \$>_{t}\right.$ as $t \rightarrow 1$, where $<f$, is an isotopy of $\mathrm{M}$ defined for $\mathrm{r}$ e $[0,1)$ and $n Q\rangle_{t}$ is flose to $p$ for all $/$ ) such that $/ ?\left(\mathrm{X}^{(1)}\right) \mathrm{nT}=0$ and $7 ?$ is $1-1$ over $M / G-T$. This forces the nondegeneracy set of $p$ to miss $\mathfrak{£}^{(1)}$ and yields $\operatorname{dem}(\mathrm{JV} />)$ $\leq 1$.

For studying the generalized manifold $M / G$ itself, the specific source manifold $M$ and the specific cell-like map $n: M \rightarrow$ A//G may not be of fundamental importance. If not, the next result indicates how to circumvent the second hypothesis in Theorem 4.1, which calls for point preimages under $n$ to have neighborhoods embeddable in SI

PROPOSITION 4.5. Ifn:M-*Yisa proper cell-like map from a 3manifold $M$ onto a generalized 3-manifold $Y$, then there exist another 3-manifold $M^{\prime}$ and a proper cell-like map $q: M^{\prime} \rightarrow Y$ such that each $<l \sim l\{y)<y G Y$, has a neighborhood in $M$ that embeds in $S^{2 \cdot}$

Proof. The argument imitates one presented in [BL] for a related result.

Theorem 1 of $[\mathrm{K}]$ helps certify that

$C=\left\{\begin{array}{lll}y & e n_{n} & l(y) \\ & \text { has no neighborhood embeddable in } S^{3}\end{array}\right\}$

is a locally finite subset of $Y$ (for the nonorientable case, see also [RL, Proposition 2.1]). The same modification will be done near each $c \mathrm{G} \mathrm{C}$, so for simplicity assume $\mathrm{C}$ consists of a single point. 
Set $P=n^{x(C)}$. By [BL, Lemma C], $P=f\left(N i\right.$, where $N_{t}$ consists of a homotopy 3-cell $Q$; with some attached 1-handles, and where $N j_{+i}$ c $N,(i=1,2, \ldots)$. Trim $Q_{t}$ by deleting an open collar on its boundary to form a smaller homotopy 3-cell $Q$, and obtain $M^{\prime}$ as the decomposition of $M$ determined by identifying $Q I$ to a point. According to [BL, Lemma D], there exist a cell-like map $q_{i} M^{\prime}{ }^{*} Y$ and a homeomorphism 6 of $M-\mathrm{ft}^{\prime \prime}{ }^{(\mathrm{C})}$ onto $\mathrm{Af}-<? \sim(\mathrm{C})$ such that

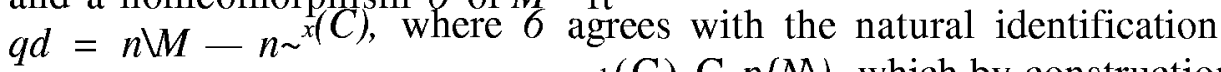
$p: M-M^{\prime}$ on $M-M$. Then ${ }^{\wedge-1}(\mathrm{C}) \mathrm{C} p(M)$, which by construction is a cube-with-handles. Application of the homeomorphism 6 quickly shows the other point preimages $q^{\sim^{x}(y)}$ to have neighborhoods embeddable in $S^{3}$ as well.

COROLLARY 4.6. If $G$ is a cell-like decomposition of a 3-manifold $M$ such that the singular set $S(M / G)$ lies in a 1 -complex topologically embedded in $M / G$ as a closed subset, then (M/Gd $\mathrm{x}$ be $F^{l}$ approximated by and the natural map $M \times E^{l} \longrightarrow(M / G) \times E^{l}{ }^{2} n^{\times}$be approximated by homeomorphisms.

Proof. To see why $(M / G) x E^{l}$ is a 4-manifold, use Proposition 4.5 to reduce to the case where each $g \in G$ has a neighborhoodempeddabde
in $\mathrm{S}^{3}$, and apply Theorem 4.1 and [DP2]. That $M \times E^{\mathrm{x}}$ (D)

can be approximated by homeomorphisms then follows from Quinn's Cell-like Approximation Theorem [Q].

COROLLARY 4.7. If $G$ is a cell-like decomposition of a 3-manifold $M$ such that the singular set $S(M / G)$ lies in a 2-complex embedded in $M / G$ as a closed subset, then the trivial extension $G^{T}$ of $G$ over $M \times E^{t}$ is shrinkable. Proof. As in Theorem 3.1, construct a homeomorphisman\$o fatiffy fing
to itself commuting with the projection $M \times E^{x} \longrightarrow E^{x}$

(1) each nondegenerate element $<£(\# *), g^{*}$ e $G^{T}$, lies in a level $M x\{t\}$ of $M x E^{x}$ and

(2) the decomposition $\&^{l}$ of $M$ induced by $\$>\left(G^{T}\right)$ on any level has its singular set, $S\left(M / \&^{1)}\right.$, confined to a 1 -complex embedded in $M / \mathscr{G}^{t}$ as a closed subset.

Again apply [DPI]. 


\section{REFERENCES}

[A] F. D. Ancel, Resolving wild embeddings in dimensions greater than 3, Topology Appl., 24 (1986), 13-40.

[BK] R. H. Bing and J. M. Kister, Taming complexes in hyperplanes, Duke Math. J., 31 (1964), 491-511.

[BL] J. L. Bryant and R. C. Lacher, Resolving acyclic images ofi-manifolds, Math. Proc. Camb. Phil. Soc, 88 (1980), 311-320.

[DI] R. J. Daverman, Products of cell-like decompositions, Topology Appl., 11 (1980), 121-139.

[D2]__ Detecting the disjoint disks property, Pacific J. Math., 93 (1981), 277 298.

[DP 1] R. J. Daverman and D. K. Preston, Shrinking certain sliced decompositions of ${ }^{\prime \prime+l,}$ Proc. Amer. Math. Soc, 79 (1980), $477-483$.

[DP2]___ Cell-like l-dimensional decompositions ofS* are 4-manifold factors, Houston J. Math., 6 (1980), 491-502.

[EI] R. D. Edwards Dimension Theory, I, in Geometric Topology (L. C. Glaser, and T. B. Rushing, Jr., editors), Lecture Notes in Math., vol. 438, Springer-Verlag, Berlin, 1975, 195-211.

[E2]__ The topology of manifolds and cell-like maps, in Proceedings of the International Congress of Mathematicians, Helsinki, 1978, Academia Scientarium Fennica, Helsinki, 1980, 111-127.

[H] D. W. Henderson, Extensions ofDehn 's Lemma and the loop theorem, Trans. Amer. Math. Soc, 120 (1965), 448-469.

[HW] W. Hurewicz and H. Wallman, Dimension Theory, Princeton Univ. Press, Princeton, N. J., 1941.

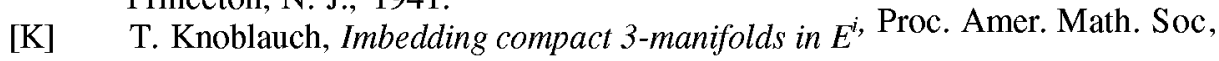
48 (1975), 447-453.

[KW] G. Kozlowski and J. J. Walsh, Cell-like mappings on 3-manifolds, Topology, 22(1983), 147-151.

[Ml] D. R. McMillan, Jr., A criterion for cellularity in a manifold, Annals of Math., 79 (1964), 327-337.

[M2]__ Canonical neighborhoods in 3-manifolds, in Topology Seminar Wisconsin, 1965 (R. H. Bing and R. J. Bean, editors), Annals of Math. Studies, vol. 60, Princeton Univ. Press, Princeton, N. J., 1966, 61-67.

[M3]__ Compact, acyclic subsets of three-manifolds, Michigan Math. J., 19 (1969), 129-136.

[Q] F- Quinn, Ends ofMaps HI: dimensions 4 and 5, J. Differential Geom., 17 (1982), 503-552.

[RL] D. Repovš and R. C. Lacher, Resolving acyclic images ofnonorientable threemanifolds, Proc. Amer. Math. Soc, 90 (1984), 157-161.

[R] W. H. Row, Jr., Compact sets definable by free 3-manifolds, Trans. Amer. Math. Soc, 197 (1974), 225-244.

[SW] A. Shapiro and J. H. C. Whitehead, A proofand extension ofDehn's lemma, Bull. Amer. Math. Soc, 64 (1958), 174-178.

[St] M. A. Stan'ko, The embedding of compacta in euclidean space, Mat. Sbornik, 83 (125) (1970), 234-255 [= Math. USSR Sbornik 12 (1970), 234-254].

[T] H. Torunczyk, On CE images of the Hilbert cube and characterizations of Q-manifolds, Fund. Math., 106 (1980), 31-40. 
[W] J. J. Walsh, The finite dimensionality ofintegral homology 3-manifolds, Proc. Amer. Math. Soc, 88 (1983), 154-156.

Received November 21, 1985 and in revised form February 1, 1988.

UNIVERSITY OF TENNESSEE

KNOXVILLE, TN 37996-1300 



\section{PACIFIC JOURNAL OF MATHEMATICS \\ EDITORS}

\author{
V. S. VARADARAJAN \\ (Managing Editor) \\ University of California \\ Los Angeles, CA 90024 \\ HERBERT CLEMENS \\ University of Utah \\ Salt Lake City, UT 84112 \\ R. FINN \\ Stanford University \\ Stanford, CA 94305
}

\author{
HERMANN FLASCHKA \\ University of Arizona \\ Tucson, AZ 85721
}

vAUGHAN F. R. JONES

University of California

Berkeley, CA 94720

STEVENKERCKH OFF

Stanford University

Stanford, CA 94305

\author{
ROBION KIRBY \\ University of California \\ Berkeley, CA 94720 \\ C. C. MOORE \\ University of California \\ Berkeley, CA 94720 \\ HAROLD T ARK
}

University of California, San Diego La Jolla, CA 92093

\section{ASSOCIATE EDITORS}
R. ARENS
E. F. BECKENBACH
B. H. NEUMANN
F. WOLF
K. YOSHIDA

(1906-1982)

\section{SUPPORTING INSTITUTIONS}

\author{
UNIVERSITY OF ARIZONA \\ UNIVERSITY OF BRITISH COLUMBIA \\ CALIFORNIA INSTITUTE OF TECHNOLOGY \\ UNIVERSITY OF CALIFORNIA \\ MONTANA STATE UNIVERSITY \\ UNIVERSITY OF NEVADA, RENO \\ NEW MEXICO STATE UNIVERSITY \\ OREGON STATE UNIVERSITY
}

\author{
UNIVERSITY OF OREGON \\ UNIVERSITY OF SOUTHERN CALIFORNIA \\ STANFORD UNIVERSITY \\ UNIVERSITY OF HAWAII \\ UNIVERSITY OF TOKYO \\ UNIVERSITY OF UTAH \\ WASHINGTON STATE UNIVERSITY \\ UNIVERSITY OF WASHINGTON
}

The Supporting Institutions listed above contribute to the cost of publication of this Journal, but they are not owners or publishers and have no responsibility for its content or policies.

Mathematical papers intended for publication in the Pacific Journal of Mathematics should be in typed form or offset-reproduced (not dittoed), double spaced with large margins. Please do not use built up fractions in the text of the manuscript. However, you may use them in the displayed equations. Underline Greek letters in red, German in green, and script in blue. The first paragraph must be capable of being used separately as a synopsis of the entire paper. In particular it should contain no bibliographic references. Please propose a heading for the odd numbered pages of less than 35 characters. Manuscripts, in triplicate, may be sent to any one of the editors. Please classify according to the scheme of Math. Reviews, Index to Vol. 39. Supply name and address of author to whom proofs should be sent. All other communications should be addressed to the managing editor, or Elaine Barth, University of California, Los Angeles, California 90024.

There are page-charges associated with articles appearing in the Pacific Journal of Mathematics. These charges are expected to be paid by the author's University, Government Agency or Company. If the author or authors do not have access to such Institutional support these charges are waived. Single authors will receive 50 free reprints; joint authors will receive a total of 100 free reprints. Additional copies may be obtained at cost in multiples of 50 .

The Pacific Journal of Mathematics is issued monthly as of January 1966. Regular subscription rate: $\$ 190.00$ a year (5 Vols., 10 issues). Special rate: $\$ 95.00$ a year to individual members of supporting institutions.

Subscriptions, orders for numbers issued in the last three calendar years, and changes of address should be sent to Pacific Journal of Mathematics, P.O. Box 969, Carmel Valley, CA 93924, U.S.A. Old back numbers obtainable from Kraus Periodicals Co., Route 100, Millwood, NY 10546.

The Pacific Journal of Mathematics at P.O. Box 969, Carmel Valley, CA 93924 (ISSN 0030-8730) publishes 5 volumes per year. Application to mail at Second-class postage rates is pending at Carmel Valley, California, and additional mailing offices. Postmaster: send address changes to Pacific Journal of Mathematics, P.O. Box 969, Carmel Valley, CA 93924.

\section{PUBLISHED BY PACIFIC JOURNAL OF MATHEMATICS, A NON-PROFIT CORPORATION} Copyright (C) 1988 by Pacific Journal of Mathematics 


\section{Pacific Journal of Mathematics}

Vol. 135, No. $2 \quad$ October, 1988

Waleed A. Al-Salam and Mourad Ismail, $q$-beta integrals and the

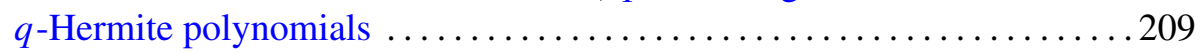

Johnny E. Brown, On the Ilieff-Sendov conjecture $\ldots \ldots \ldots \ldots \ldots \ldots \ldots 223$

Lawrence Jay Corwin and Frederick Paul Greenleaf, Spectrum and

multiplicities for restrictions of unitary representations in nilpotent Lie

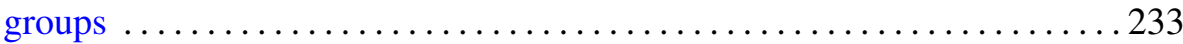

Robert Jay Daverman, 1-dimensional phenomena in cell-like mappings on 3-manifolds .......................................... 269

P. D. T. A. Elliott, A localized Erdős-Wintner theorem .............. 287

Richard John Gardner, Relative width measures and the plank problem . . . 299

F. Garibay, Peter Abraham Greenberg, L. Reséndis and Juan José

Rivaud, The geometry of sum-preserving permutations ............313

Shanyu Ji, Uniqueness problem without multiplicities in value distribution

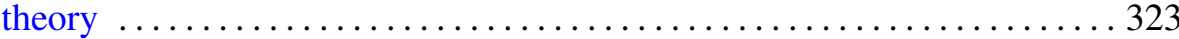

Igal Megory-Cohen, Finite-dimensional representation of classical

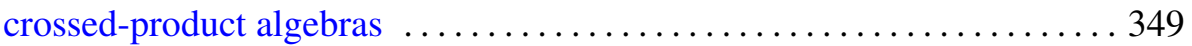

Mirko Navara, Pavel Pták and Vladimír Rogalewicz, Enlargements of

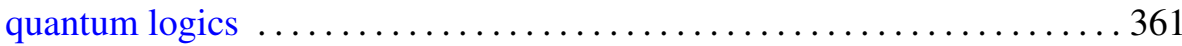

Claudio Nebbia, Amenability and Kunze-Stein property for groups acting

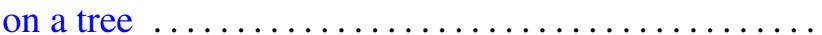

Chull Park and David Lee Skoug, A simple formula for conditional Wiener

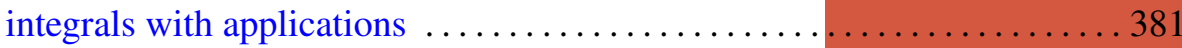

Ronald Scott Irving and Brad Shelton, Correction to: "Loewy series and simple projective modules in the category $\mathrm{O}_{s} " \ldots .$.

Robert Tijdeman and Lian Xiang Wang, Correction to: "Sums of products

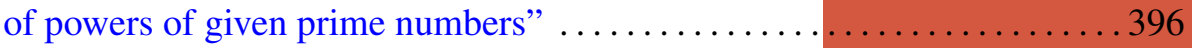

